\title{
Câncer infantojuvenil: relação com os polos de irrigação agrícola no estado do Ceará, Brasil
}

\author{
Cancer among children and adolescents: relationship \\ with the poles of agricultural irrigation in the state of Ceará, Brazil
}

Isadora Marques Barbosa (https://orcid.org/0000-0002-3799-7882) ${ }^{1}$

Diane Sousa Sales (https://orcid.org/0000-0001-8709-0675) ${ }^{2}$

Miren Uribe Arregi (https://orcid.org/0000-0002-3839-8056) ${ }^{3}$

Raquel Maria Rigotto (https://orcid.org/0000-0002-7460-3221) ${ }^{1}$

${ }^{1}$ Departamento de Saúde Comunitária, Centro de Ciências da Saúde, Universidade Federal do Ceará. R. Alexandre Baraúna, Rodolfo Teófilo. 60430-160 Fortaleza CE Brasil.

isadoramarx@gmail.com

${ }^{2}$ Universidade Estadual do Ceará. Fortaleza CE Brasil.

${ }^{3}$ Instituto do Câncer do

Ceará. Fortaleza CE Brasil.

\begin{abstract}
The scope of this article is to assess the magnitude and the spatial and temporal trend of cancer in children and adolescents and its association with the agricultural irrigation poles in the State of Ceará from 2000 to 2012. This involves ecological research on morbidity and mortality indicators in the State Ceará between 2000 and 2012. The study population was made up of children and adolescents aged from 0 to 19 years diagnosed with cancer. Information was collected in the Hospital Cancer Register and in the Mortality Information System. A total of 3274 cases were treated in CACONs and UNACONs and 2,080 deaths due to neoplasms were identified. In the association of cancer cases with agricultural irrigation poles, it was detected that the highest concentrations of cases are in micro-regions that have irrigation poles. The microregions with the highest concentrations of child and adolescent cancer coincide with the agricultural irrigation poles.
\end{abstract}

Key words Cancer, Child, Adolescent, Agrochemicals
Resumo O objetivo deste artigo é avaliar a magnitude, a tendência espacial e temporal do câncer infantojuvenil e sua associação com os polos de irrigação agrícola, no estado do Ceará, de 2000 a 2012. Pesquisa ecológica sobre os indicadores de morbidade e mortalidade do estado do Ceará de 2000 a 2012. População do estudo foi composta por crianças e adolescentes com idade de 0 a 19 anos e com diagnóstico de câncer. Foram coletadas informações no Registro Hospitalar de Câncer e no Sistema de Informação sobre Mortalidade. Foram atendidos 3.274 casos em CACONs e UNACONs e 2.080 óbitos por neoplasias. Na associação dos casos de câncer com os polos de irrigação agrícola, percebeu-se que as maiores concentrações de casos estão nas microrregiões que apresentam polos de irrigação. As microrregiões com maiores concentrações de casos de câncer infantojuvenil coincidem com os polos de irrigação agrícola. Palavras-chave Câncer, Criança, Adolescente, Agroquímicos 


\section{Introdução}

De acordo com a Agência de Desenvolvimento do Ceará (ADECE) ${ }^{1}$, este estado iniciou, nos anos 1970, um amplo programa de agricultura irrigada a partir da implantação de perímetros públicos irrigados. O Programa Cearense de Agricultura Irrigada (PROCEAGRI) foi criado para incentivar a produção de frutas, flores e hortaliças, tendo sido necessário eleger polos de produção que tivessem potencial para irrigação das plantações. De acordo com a ADECE ${ }^{1}$ o estado do Ceará apresenta seis polos de irrigação, sendo eles: Baixo Acaraú, Baixo Jaguaribe, Cariri, Centro Sul, Ibiapaba e Metropolitano.

O modelo produtivo monocultor do agronegócio exige a utilização intensiva de agrotóxicos, a qual compromete o ambiente, estando o mesmo inter-relacionado com a saúde humana. O aumento considerável no consumo de agrotóxicos utilizados tem ocasionado uma série de transtornos e alterações ao ambiente, seja pela contaminação das comunidades de seres vivos, quanto pela acumulação nos segmentos bióticos e abióticos do ecossistema².

O INCA (Instituto Nacional de Câncer), na publicação Vigilância do Câncer Relacionado ao Trabalho e ao Ambiente ${ }^{3}$, afirma que a exposição aos agrotóxicos pode ser considerada como uma das condições potencialmente associadas ao desenvolvimento do câncer.

Uma revisão sistemática ${ }^{4}$ sobre o câncer infantojuvenil e o uso de agrotóxico evidenciou uma associação entre a exposição mais longa e com maiores doses de agrotóxicos e o aparecimento de leucemia e linfoma não Hodgkin. Esse mesmo estudo pôde concluir que medidas para evitar a exposição a agentes agrotóxicos são relevantes para a prevenção do câncer. Estudo de Curvo et al..$^{5}$ conclui que a exposição aos agrotóxicos desde o nascimento mostra-se relevante como indicador de morbidade e mortalidade por câncer em menores de 20 anos.

Para avaliar a associação dos agentes agrotóxicos e correlacionar com seu potencial efeito carcinogênico, algumas dificuldades são encontradas, como: a ausência de dados adequados sobre a natureza da exposição, a diversidade de ingredientes ativos utilizados, as mudanças constantes dos produtos utilizados, a diversidade de formas de utilização desses produtos, o longo período necessário para o surgimento do câncer e as dificuldades na obtenção de dados sobre exposições ambientais ${ }^{6}$.
As neoplasias na infância e na adolescência são consideradas as principais causas de morte por doenças e as mesmas sofrem grande influência dos fatores de risco ambientais na sua etiologia. Alguns desses fatores são conhecidos, mas poucos são os estudos que buscam uma avaliação para o surgimento de neoplasias, como é o caso da exposição ao agrotóxico como fator de risco, por exemplo, para as leucemias e linfoma não-Hodgkin, mas pouco abordados, de maneira especial na população infantojuvenil.

O objetivo desta pesquisa foi avaliar a magnitude, a tendência espacial e temporal do câncer infantojuvenil, e sua associação com os polos de irrigação agrícola no estado do Ceará de 2000 a 2012.

\section{Métodos}

Realizou-se uma pesquisa analítica com delineamento ecológico de séries históricas das informações sobre os indicadores de morbidade e mortalidade do estado do Ceará de 2000 a 2012. Considerou-se a população de indivíduos com faixa etária entre 0 e 19 anos de idade e com diagnóstico de câncer.

\section{Local da pesquisa}

O estado apresenta 184 municípios, divididos em 33 microrregiões do Instituto Brasileiro de Geografia e Estatística (IBGE) ${ }^{6}$. Com relação à agricultura, considerou-se a divisão territorial a partir de polos de irrigação implantados em alguns municípios. De acordo com a ADECE, o estado do Ceará apresenta seis polos de irrigação, totalizando 64 municípios ${ }^{1}$.

\section{Coleta dos dados}

Os seguintes itens do Registro Hospitalar de Câncer (RHC) foram utilizado para a coleta dos dados sobre os casos de câncer infantojuvenil: idade, sexo, local de residência, tipo histológico, ano da primeira consulta e unidade hospitalar de atendimento.

Em relação à mortalidade por câncer infantojuvenil, os dados foram obtidos do Sistema de Informação sobre Mortalidade (SIM) no período de 2000 a 2012. Nesse sistema foram coletados os seguintes dados de crianças e adolescentes na faixa-etária de 0 a 19 anos de idade: sexo, capítulo II - Neoplasias (tumores) da CID 10, local de residência e microrregião do IBGE. 
itiram calcular a incidência: tipo histológico, sexo e grupo de idade. Essa análise foi realizada por microrregião e para o estado, com base no local de residência dos casos, no período de 2000 a 2011. O mapeamento dos casos registrados foi realizado no tabwin versão $3.6 \mathrm{~b}$ de 2010 .

O cálculo do coeficiente de mortalidade ajustado por idade foi obtido a partir da razão entre o número de óbitos por causa básica do óbito (de acordo com o capítulo II da CID 10), por microrregião ou estado, em um determinado período, pela população exposta de acordo com os censos de 2000, 2010 e pelas projeções populacionais encontradas no Datasus, envolvendo 1.000 .000 de habitantes. O mapeamento dos casos registrados foi realizado no tabwin versão 3.6b de 2010 .

Para estimar a variação anual percentual e identificar pontos em que há modificação da tendência na mortalidade, para as microrregiões de saúde estudadas, foi utilizado o programa Joinpoint versão 3.5.1 (Statistical Researchand Applications Branch, National Cancer Institute, Rockville, MD, EUA) de modo a executar a regressão linear segmentada (joinpoint regression).

A ideia inicial para a coleta dos dados da pesquisa seria utilizar registros sobre o consumo de agrotóxicos no estado do Ceará, por município. Entretanto, após incessantes buscas por essas informações em órgãos como a Agência de Defesa Agropecuária do Estado do Ceará (ADAGRI) e a Diretoria Florestal, o setor de agrotóxicos da Superintendência Estadual do Meio Ambiente (SEMACE), nos foi informado que não existem dados sobre consumo de agrotóxicos no estado, o que dificultou a escolha metodológica. A estratégia utilizada, por falta de dados sobre consumo de agrotóxicos, foi de comparar a distribuição espacial por microrregião do IBGE dos casos e das taxas de mortalidade por câncer infantojuvenil com as delimitações espaciais dos polos de irrigação do estado do Ceará.

Este estudo foi realizado de acordo com a Resolução 466/2012, a qual regulamenta diretrizes e normas das pesquisas envolvendo seres humanos. Obteve parecer favorável, pelo Comitê de Ética em Pesquisa (CEP).

\section{Resultados}

\section{Casos de câncer infantojuvenil no estado do Ceará}

De acordo com o RHC, no período de 2000 a 2011 foram registrados 3.274 casos de câncer infantojuvenil. Salientamos que esse número não representa a quantidade de casos novos, mas todos os que deram entrada, pela primeira vez durante o período, na unidade de tratamento para realizar algum tipo de acompanhamento.

Do total de registros encontrados para esse período, $1.806(55,2 \%)$ eram do sexo masculino. Ao analisar por faixa etária, nota-se que $26,3 \%$ dos casos apresentam idade entre 15 a 19 anos, seguido de $23,7 \%$ na faixa etária de 10 a 14 anos, $23,2 \%$ com idade entre 1 e 4 anos, $22,5 \%$ entre 5 a 9 anos e 4,2\% com menos de 1 ano de idade.

Com relação ao tipo histológico do câncer, evidencia-se que, do total de registros no período de 2000 a 2011, 29,5\% foram de leucemias, $16 \%$ de linfomas $(8,4 \%$ de linfoma de Hodgking e 7,6\% de linfomas não-Hodgking) e 12,6\% de câncer no sistema nervoso central (SNC) (com localização nas meninges, encéfalo e medula espinhal e outras partes do SNC) e $41,9 \%$ por outros tipos histológicos.

Pela análise por microrregião do estado do Ceará no período de 2000 a 2011, a partir do total de casos registrados nesse período, encontrou-se que as quatro com mais casos de câncer foram: Fortaleza (49,9\%), Sobral (4,0\%), Baixo Jaguaribe $(3,7 \%)$ e Cariri $(3,5 \%)$.

\section{Mortalidade por câncer infantojuvenil no estado do Ceará}

De acordo com o Sistema de Informação sobre Mortalidade (SIM), no período de 2000 a 2012 foram registrados 2080 óbitos por neoplasias no estado do Ceará, na população com menos de 20 anos de idade. Destes, 1.211 (58,2\%) ocorridos no sexo masculino. A média das taxas de mortalidade no período de 2000 a 2012 para a população de 0 a 19 anos de idade foi de 48 óbitos por 1.000.000 de habitantes.

Quanto à faixa etária dos óbitos por câncer, de acordo com o número total de registros no período de 2000 a 2012, a mais acometida foi a de 15 a 19 anos, com $28,4 \%$ dos casos, seguida pela de 5 a 9 anos $(23,2 \%)$, de 10 a 14 anos (22,1\%), de 1 a 4 anos (20,5\%) e, por fim, os indivíduos com menos de 1 ano de idade (5,8\%). 
Com relação à causa básica dos óbitos registrados no período de 2000 a 2012, evidenciase que as leucemias foram as mais frequentes $(31,7 \%)$, seguidas pelas neoplasias do sistema nervoso central (18,6\%), linfomas não-Hodgkin (7\%) e neoplasia de traqueia, brônquios e pulmões $(2,1 \%)$.

Somando todos os casos no período de 2000 a 2012 e fazendo uma análise por microrregião, nota-se que as cinco com maiores coeficientes de mortalidade por 1 milhão de habitantes são: Ibiapaba (63 óbitos/1 milhão de habitantes), Várzea Alegre (57 óbitos/1 milhão de habitantes), Sertão de Inhamuns e Meruoca, cada uma com taxa de mortalidade de 54 óbitos/1 milhão de habitantes e Pacajus (53 óbitos/1 milhão de habitantes).

Ao realizar a análise de regressão joinpoint (ponto de inflexão) tendo como variáveis as taxas de mortalidade por câncer infantojuvenil nas microrregiões de saúde do estado do Ceará no período de 2000 a 2012, destaca-se a evolução das porcentagens de causas mal definidas. Esta poderia influenciar a tendência das taxas, pois foi observado que quatro microrregiões apresentaram resultados significativos para o teste de tendência (Tabela 1).

$\mathrm{Na}$ análise de regressão joinpoint (ponto de inflexão), nota-se que a microrregião de Uruburetama foi a única que apresentou redução na tendência das taxas de mortalidade para câncer infantojuvenil. As microrregiões de Camocim/ Acaraú, Baixo Jaguaribe e Cariri apresentaram aumento na média anual. Ressalta-se que essas microrregiões foram as que apresentaram uma evolução na porcentagem de causas mal definidas mais constantes e com menores porcentagens, o que indica que o aumento não vem da mudança na qualidade do Sistema de Notificação

Tabela 1. Estimativas de tendência temporal das taxas de mortalidade para câncer infantojuvenil, obtidas através de regressão joinpoint, para as microrregiões de saúde do estado do Ceará, 2000 a 2012. Fortaleza - CE, 2016.

\begin{tabular}{lrrrr}
\hline & $\begin{array}{c}\text { \% mudança } \\
\text { média anual }\end{array}$ & \multicolumn{2}{c}{$\begin{array}{c}\text { Intervalo de } \\
\text { confiança }\end{array}$} & $\begin{array}{c}\text { Valor } \\
\text { Microrregião }\end{array}$ \\
\cline { 2 - 5 } & AAPC & \multicolumn{1}{c}{ LI } & \multicolumn{1}{c}{ LS } & P \\
\hline Camocim/Acaraú & 5,2 & 1,6 & 8,8 & 0,0 \\
Uruburetama & $-35,3$ & $-57,5$ & $-1,5$ & 0,0 \\
Baixo Jaguaribe & 16,8 & 12,4 & 21,3 & 0,0 \\
Cariri & 4,8 & 1,1 & 8,8 & 0,0 \\
\hline AAPC: Average annual percent change (variação percentual anual \\
média); LI: Limite inferior; LS: Limite superior. \\
Fonte: Dados da pesquisa.
\end{tabular}

sobre Mortalidade (SIM).

\section{Casos de câncer infantojuvenil, mortalidade e polos de irrigação do estado do Ceará}

Quando a representação espacial dos registros hospitalares no estado do Ceará por microrregiões de saúde é comparada com os seis pólos de irrigação, nota-se que as microrregiões com maiores concentrações de casos de câncer infantojuvenil coincidem com estes, como ilustrado nos mapas (Figura 1).

Ressaltamos que foi necessário ocultar os dados referentes à microrregião de Fortaleza, pois a escala do mapa ficaria bastante destoante, visto que esta microrregião apresenta uma concentração de $36,7 \%$ da população total do estado do Ceará, além do fato de haver um problema na notificação de procedência dos casos, os quais muitas vezes são registrados como de Fortaleza, mesmo quando procedentes de municípios do interior do estado.

Destacam-se algumas microrregiões com altas concentrações de casos de câncer infantojuvenil, como Sobral, Ibiapaba, Baixo Jaguaribe e Cariri, com 9 a 11 casos registrados; Litoral de Acaraú e Camocim, Sertão de Quixeramobim e Iguatu, com 7 a 9 casos.

Percebe-se no mapa, que as microrregiões com maiores concentrações de casos de câncer infantojuvenil têm polos de irrigação, como a microrregião de Sobral, litoral de Acaraú e Camocim que apresentam o polo de irrigação do Baixo Acaraú. A microrregião de Ibiapaba tem o polo de irrigação de mesmo nome. As microrregiões de Baturité, Sertão de Quixeramobim e principalmente a do Baixo Jaguaribe são atingidas pelo polo de irrigação do Baixo Jaguaribe, já a de Iguatu é pelo polo de irrigação do Centro-Sul e a microrregião do Cariri pelo do Cariri.

Fazendo uma representação espacial das taxas de mortalidade no período de 2000 a 2012 nas microrregiões de saúde do estado do Ceará, e comparando com os polos de irrigação, nota-se que as microrregiões com maiores taxas de mortalidade coincidem com estes, como observado no mapa da Figura 2.

Percebe-se que as microrregiões com maiores taxas de mortalidade para o período foram Ibiapaba e Várzea Alegre, seguidos por Sobral, Meruoca, Fortaleza, Iguatu, Cariri e Sertão de Inhamuns.

Comparando essas microrregiões com os polos de irrigação, nota-se que estes estão presente nas de Ibiapaba, Sobral, Meruoca, Fortaleza, 


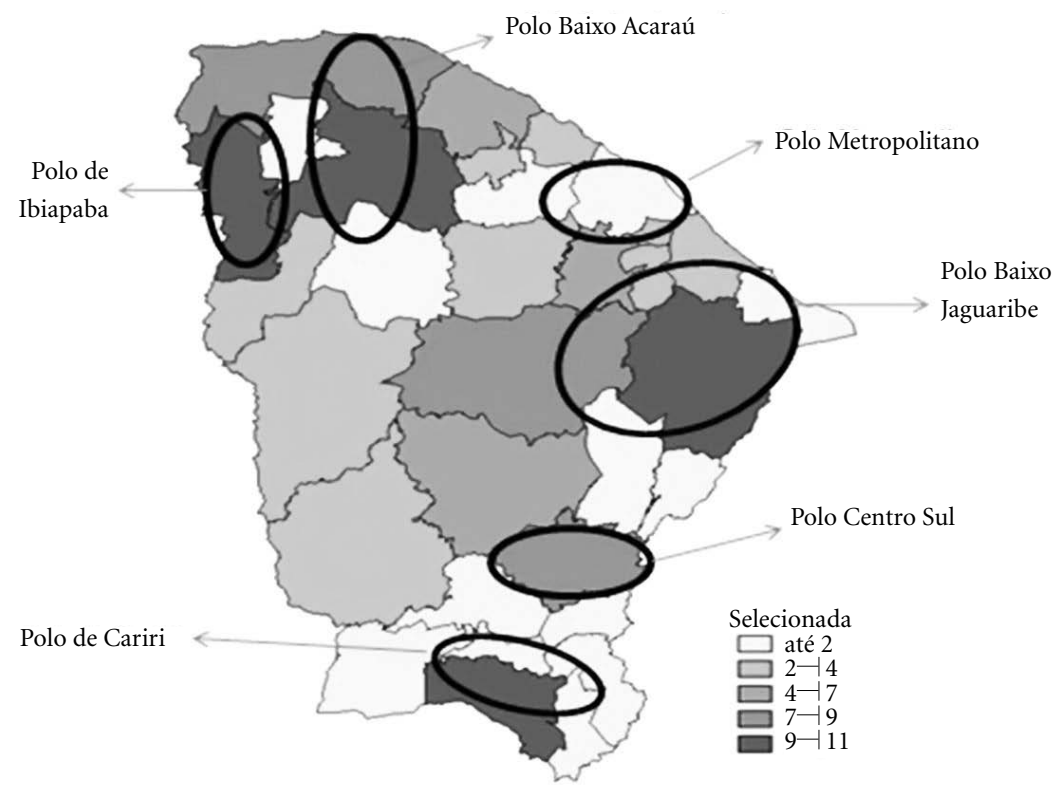

Figura 1. Distribuição espacial dos polos de irrigação e dos casos de câncer infantojuvenil por microrregião do IBGE no período de 2000 a 2011, no estado do Ceará.

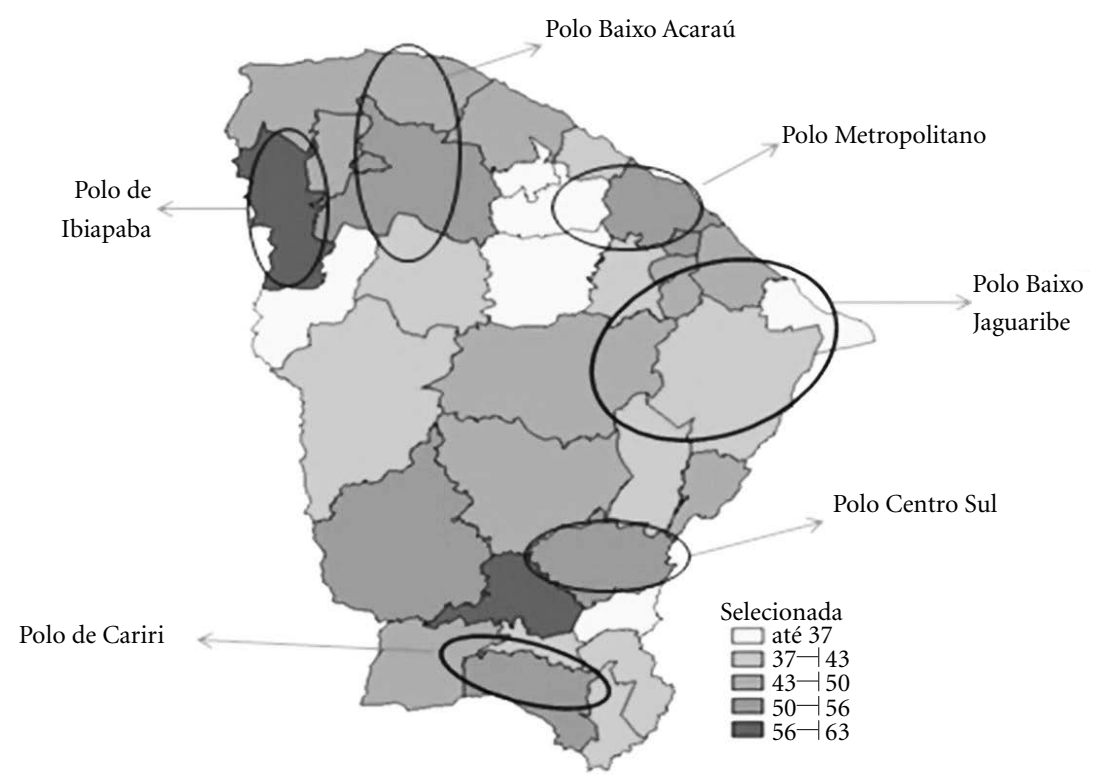

Figura 2. Distribuição espacial dos polos de irrigação e das taxas de mortalidade de câncer infantojuvenil por microrregião de saúde no período de 2000 a 2012, no estado do Ceará. 
Iguatu e Cariri. A microrregião de Várzea Alegre é cercada pelos polos do Cariri e Centro-Sul.

\section{Discussão}

No presente estudo a maioria dos casos de câncer infantojuvenil ocorreu no sexo masculino, semelhante ao encontrado no estudo de Curvo et al. ${ }^{5}$, no qual $53,7 \%$ dos registros analisados eram do sexo masculino. A faixa etária mais frequente foi a de 15 a 19 anos de idade, diferente do encontrado no estudo de Camargo et al. ${ }^{7}$, no qual a faixa etária de 1 a 4 anos apresentou a maior taxa de incidência para casos de câncer infantojuvenil. Acreditase que a divergência na faixa etária é explicada por terem sido medidas de cálculo diferentes, no presente estudo se tratou de frequência e na pesquisa de Camargo et al. ${ }^{7}$ de incidência, além das bases de dados terem sido diferentes, RHC e Registro de Câncer de Base Populacional, respectivamente.

Foi evidenciado que os tipos histológicos mais frequentes foram as leucemias, linfomas e tumores no sistema nervoso central. $\mathrm{O}$ estudo realizado por Ward et al. ${ }^{8}$ encontrou que o tipo de câncer mais frequente em crianças foram as leucemias linfoide aguda (LLA), seguidas pelos tumores no sistema nervoso central (SNC) e linfoma não-Hodgkin (LNH). Se no presente estudo a avaliação para linfomas foi realizada separadamente (LH e LNH), os tumores do SNC ocupam a segunda posição de maior frequência, corroborando, assim, o evidenciado no estudo de Ward et al. ${ }^{8}$.

As microrregiões que apresentaram mais casos de câncer infantojuvenil foram Fortaleza, Sobral, Baixo Jaguaribe e Cariri. Sabe-se que Fortaleza, Sobral e Barbalha (Cariri) são sedes de macrorregiões para tratamento do câncer e que alguns pacientes informam endereço de procedência nessas sedes, mesmo sendo de outros municípios. Entretanto, este não seria o caso do Baixo Jaguaribe, o que pode significar que esse número de casos registrados com procedência nessa microrregião, realmente seja de lá, onde desde os anos 2000 estão instaladas grandes empresas transnacionais de fruticultura irrigada para exportação, com uso intensivo de agrotóxicos, os quais, somados aos casos que forneceram o endereço de Fortaleza, sugerem que o número seja maior do que os $3,7 \%$ identificados nesse estudo.

O padrão das taxas de mortalidade por grupo etário varia para os diferentes grupos de diagnóstico. As taxas de mortalidade para todas as neoplasias costumam ser maiores em crianças com idade entre 1 e 4 anos e menores naquelas com idade abaixo de $1^{9}$.

As leucemias representaram a causa básica mais frequente no período estudado, seguidas pelas neoplasias no sistema nervoso central e linfomas não-Hodgkin. No estudo realizado em Mato Grosso, no período de 2000 a 2006, também foi evidenciado que as leucemias representaram a principal causa básica de óbito por câncer em crianças e adolescentes, seguida pelos tumores do SNC e pelos $\mathrm{LNH}^{5}$.

De acordo com Braga et al. ${ }^{10}$, a tendência na mortalidade infantil por neoplasias malignas muitas vezes pode ser explicada pela tendência da mortalidade por leucemias. Nota-se que os coeficientes de mortalidade para as leucemias apresentaram um padrão aparentemente cíclico, assim como no estudo de Ferreira9, o que poderia ser explicado pela associação das leucemias com as doenças infecciosas, entretanto para tal afirmação seriam necessários estudos com séries históricas mais longas.

As microrregiões de Baixo Jaguaribe, Camocim/Acaraú e Cariri, que tiveram aumento na tendência das taxas de mortalidade na análise de regressão joinpoint, apresentam polos de irrigação, $\mathrm{o}$ que está bastante ligado à utilização de tecnologias para o aumento na produção de alimentos (que na verdade são commodities), tais como fertilizantes e agrotóxicos. De acordo com o livro Agrotóxicos, trabalho e saúde ${ }^{11}$, de 2002 a 2012 foram despejados mais de 4.000 .000 litros de agrotóxicos na região do Baixo Jaguaribe, apenas por pulverização aérea da monocultura da banana.

Em um estudo epidemiológico realizado em áreas expostas à contaminação ambiental por agrotóxicos na região no Baixo Jaguaribe, após serem colhidas 24 amostras de água em diversas comunidades e em diferentes pontos como canal de irrigação, poços, caixas d'água residenciais, água oriunda do sistema de tratamento de uma localidade, foram encontrados agrotóxicos em $100 \%$ das amostras, totalizando 21 ingredientes ativos, inclusive com classificação toxicológica I (extremamente tóxicos) ${ }^{12}$.

No estudo realizado por Rigotto et $a . .^{13}$, na região do Baixo Jaguaribe, foi evidenciado que a taxa de internação por neoplasia foi 1,76 vezes maior quando comparado com onze municípios tidos como controle na pesquisa. Também foi evidenciado que a taxa de mortalidade por câncer é 38\% maior nos municípios do Baixo Jaguaribe.

O estudo realizado por Bassil et al..$^{14}$ buscou rever na literatura o que foi documentado sobre o uso de agrotóxicos e o câncer. Por essa revisão 
sistemática, existe uma relação positiva entre a exposição a agrotóxicos e o desenvolvimento de alguns tipos de câncer, especialmente no cérebro, na próstata, nos rins, o linfoma não-Hodgkin e a leucemia. Jobim et al. ${ }^{15}$, buscando contribuir no debate sobre a associação entre mortalidade por câncer e uso de agrotóxicos, concluiu que não se pode descartar a influência de exposições a agrotóxicos como um fator contribuinte à maior taxa de mortalidade por neoplasias.

\section{Conclusão}

Identificou-se que a faixa etária com maiores números de óbitos registrados de câncer infantojuvenil foi a de 15 a 19 anos de idade. Os coeficientes de mortalidade para as leucemias foram os maiores evidenciados no período de 2000 a 2012. As microrregiões de saúde com maiores taxas de mortalidade foram Ibiapaba, Várzea Alegre, Sertão de Inhamuns e Meruoca. Na análise da tendência temporal das taxas de mortalidade para câncer infantojuvenil verificou-se que Camocim/ Acaraú, Baixo Jaguaribe e Cariri apresentaram aumento na média anual no período estudado.

$\mathrm{Na}$ associação dos casos de câncer com os polos de irrigação agrícola, evidenciou-se que as maiores concentrações de casos estão nas microrregiões que apresentam polos de irrigação. $\mathrm{Na}$ distribuição das taxas de mortalidade para câncer infantojuvenil no estado do Ceará, notou-se que as microrregiões de Ibiapaba, Sobral, Meruoca, Fortaleza e Cariri, que apresentaram altas taxas de mortalidade, também apresentam polos de irrigação.
Embora limitadas, as evidências de associação entre a exposição a agrotóxicos, por meio da análise por polos de irrigação, e o câncer infantojuvenil são suficientes para indicar a necessidade de promoção de tecnologias e políticas agrícolas que não dependam do uso desses defensivos, como a Agroecologia. Dentro do arcabouço jurídico-institucional que delineia as políticas de saúde pública, faz-se urgente ampliar o diagnóstico dos efeitos crônicos dos agrotóxicos, especialmente os cânceres; aperfeiçoar sua notificação e implementar efetivamente a vigilância da saúde.

O Sistema Único de Saúde deveria investir em capacitações para a melhoria dos registros nos sistemas de notificação, vigilância sobre a história ocupacional e ambiental dos casos notificados e incentivar ações de educação em saúde que visem reduzir os fatores de riscos associados à carcinogênese. Destaca-se como ação importante a preservação e o fomento da agricultura camponesa, sem uso de agrotóxicos, considerando esta forma a mais segura para o ambiente e para a saúde humana.

A pesquisa apresentou como limitação a incerteza sobre a fidedignidade dos registros de câncer, quando se trata de procedência e a inexistência de dados referentes ao consumo de agrotóxicos, por município, no estado do Ceará, visto que os dados oficiais disponíveis são sobre comercialização e não representam o consumo, o que diminui a escolha de estratégias metodológicas para a investigação, contribuindo para a invisibilização dos efeitos crônicos ocasionados por exposições a agrotóxicos.

\section{Colaboradores}

IM Barbosa, MU Arregi e RM Rigotto: participação em todas as etapas. DS Sales: Participação na análise dos resultados, elaboração da discussão e considerações finais. 


\section{Referências}

1. Agência de Desenvolvimento do Ceará (ADECE). Perfil da produção de frutas. Brasil, Ceará, 2013. [acessado 2015 Jan 20]. Disponível em: http://www.adece.ce.gov. br/phocadownload/Agronegocio/perfil_da_producao_ de_frutas_brasil_ceara_2013_frutal.pdf

2. Ribas PP, Matsumura ATS. A química dos agrotóxicos: impacto sobre a saúde e meio ambiente. Revista Liberato $2009 ; 10(14): 149-158$.

3. Instituto Nacional de Câncer (INCA). Vigilância do Câncer relacionado ao trabalho e ao ambiente. $2^{\mathrm{a}} \mathrm{ed}$. rev. atual. Rio de Janeiro: INCA; 2010.

4. Sanborn M, Kerr KJ, Sanin LH, Cole DC, Bassil KL, Vakil C. Non-cancer Health effects of pesticides systematic review and implications for family doctors. Can Fam Physician 2007; 53(10):1712-1720.

5. Curvo HRM, Pignati WA, Pignatti MG. Morbimortalidade por câncer infantojuvenil associada ao uso agrícola de agrotóxicos no estado de Mato Grosso, Brasil. Cad Saúde Colet 2013; 21(1):10-17.

6. Instituto Brasileiro de Geografia e Estatística (IBGE). Pesquisa Agrícola Municipal. Rio de Janeiro: IBGE. [acessado 2016 Ago 20]. Disponível em: http://www.sidra.ibge.gov.br/bda/acervo/acervo9.asp? $=c \& p=P A \&-$ $\mathrm{z}=\mathrm{t} \& \mathrm{o}=11$

7. Camargo B, Santos MO, Rebelo MS, Reis RS, Ferman S, Noronha CP, Pombo-de-Oliveira MS. Cancer incidence among children and adolescents in Brazil: first report of 14 population-based cancer registries. Int $\mathrm{J}$ Cancer 2010; 126(3):715-720.

8. Ward E, Desantis C, Robbins AMD, Kohler B, Jemal ADVM. Childhood and adolescent cancer statistics, 2014. Cancer J Clin 2014; 64(2):83-103

9. Ferreira JMO. Incidência, mortalidade e sobrevida de leucemia e linfoma no município de Fortaleza, Ceará [dissertação]. Rio de Janeiro: Fiocruz; 2010.
10. Braga PE, Latorre MRDO, Curado MP. Câncer na infância: análise comparativa da incidência, mortalidade e sobrevida em Goiânia (Brasil) e outros países. Cad Saude Publica 2002; 18(1):33-44.

11. Rigotto RM, organizadora. Agrotóxicos, trabalho e saúde: vulnerabilidade e resistência no contexto da modernização agrícola no baixo Jaguaribe/CE. Fortaleza: Edições UFC, Expressão Popular; 2011.

12. Rigotto RM, Pessoa VM. Estudo epidemiológico da população da região do baixo Jaguaribe exposta à contaminação ambiental em área de uso de agrotóxicos Documento sintese dos resultados parciais da pesquisa. Fortaleza: UFCE; agosto de 2010.

13. Rigotto RM, Silva AMC, Ferreira MJM, Rosa IF, Aguiar ACP. Tendências de agravos crônicos à saúde associados a agrotóxicos em região de fruticultura no Ceará, Brasil. Rev Bras Epidemiol 2013; 16(3):763-773.

14. Bassil KL, Vakil C, Sanborn M, Cole DC, Kaur JS, Kerr KJ. Cancer health effects of pesticides: systematic review. Can Fam Physician 2007; 53(10):1704-1711.

15. Jobim PFC, Nunes LN, Giugliani R, Cruz IBM. Existe uma associação entre mortalidade por câncer e uso de agrotóxicos? Uma contribuição ao debate. Cien Saude Colet 2010; 15(1):277-288.

Artigo apresentado em 17/03/2017

Aprovado em 26/07/2017

Versão final apresentada em 28/07/2017 